\title{
Transfert : les déterminants de la performance des exploitations agricoles familiales
}

Quebec: Financial and technical-economic components of farms performance after intergenerational transfer

Fanny Lepage, Jean Pierre Couderc, Jean-Philippe Perrier et Diane Parent

\section{OpenEdition}

Journals

Édition électronique

URL : http://journals.openedition.org/economierurale/3058

DOI : 10.4000/economierurale.3058

ISSN : 2105-2581

Éditeur

Société Française d'Économie Rurale (SFER)

Édition imprimée

Date de publication : 30 juillet 2011

Pagination : 3-17

ISSN : 0013-0559

Référence électronique

Fanny Lepage, Jean Pierre Couderc, Jean-Philippe Perrier et Diane Parent, «Transfert : les déterminants de la performance des exploitations agricoles familiales », Économie rurale [En ligne], 324 | juillet-août 2011, mis en ligne le 30 juillet 2013, consulté le 30 avril 2019. URL : http:// journals.openedition.org/economierurale/3058 ; DOI : 10.4000/economierurale.3058 


\section{Québec: la performance des exploitations agricoles à la suite du transfert intergénérationnel}

Fanny LEPAGE, Jean-Pierre COUDERC • Supagro, Marchés, organisations, institutions et stratégies d'acteurs (MOISA), Montpellier, lepage@supagro.inra.fr, couderjp@supagro.inra.fr

Jean-Philippe PERRIER • Université Laval, Département d'économie agroalimentaire et sciences de la consommation, Traget Laval, Québec, Canada, JeanPhilippe.Perrier@eac.ulaval.ca

Diane PARENT • Université Laval, Département des sciences animales, Traget Laval, Québec, Canada, Diane.Parent@fsaa.ulaval.ca

\section{Introduction}

e transfert des exploitations agricoles devient un objectif significatif de l'agriculture du $\mathrm{XXI}^{\mathrm{e}}$ siècle au Canada et dans plusieurs autres pays industrialisés. Auparavant, les exploitations agricoles n'avaient qu'une faible valeur monétaire et étaient simplement léguées à l'un des fils. Aujourd'hui, les fermes de plus en plus capitalisées et de moins en moins rentables sont difficilement transférables.

L'agriculture est un secteur économique où la majorité des exploitations reste encore de nos jours détenue et gérée par le noyau familial. Toutefois, depuis l'industrialisation de l'agriculture et encore aujourd'hui, il y a un déclin constant des exploitations : une chute de 4,5\% entre les recensements de 2001 et de 2006 au Québec (Statistique Canada, 2006). En plus de cette décroissance, l'augmentation de l'âge moyen des exploitants rend de plus en plus actuelle la question de la retraite et des transferts d'exploitation en agriculture : au Québec, il est passé de 47 ans en 2001 à 49,3 ans en 2006. L'arrivée de l'âge de la retraite dans le secteur agricole implique, soit le transfert de l'exploitation à une relève familiale, soit la vente à une personne hors cadre familial, ou encore le démantèlement de l'exploitation.

La première option, le transfert familial, représente $73 \%$ des établissements en agriculture (MAPAQ, 2006). Plusieurs contraintes augmentent la difficulté de reprendre la ferme et d'en assurer la pérennité. Le peu de rentabilité en agriculture, la faiblesse des revenus de retraite (Vilain, 2004) et la hausse des valeurs des actifs agricoles (Levallois, 2003) représentent un frein à la reprise, mais également des causes d'insuccès des transferts. D'autres éléments organisationnels nuisent au transfert tel que le manque de préparation, le désir des parents d'avoir un revenu satisfaisant à la retraite, ainsi que leur volonté d'être équitable envers tous leurs enfants. Tous ces constats incitent à se préoccuper de la réussite des transferts en se remémorant qu'à chaque échec le secteur agricole subit un dépeuplement de ses territoires, sans compter les implications sur la durabilité de l'agriculture familiale versus l'agriculture industrielle et salariée (Lobley, 2007).

La problématique du transfert des exploitations agricoles familiales attire de plus en plus l'attention des chercheurs. Les principaux sujets étudiés ont été les facteurs de succès et d'insuccès des transferts (Eaton, 1993 ; Parent et al., 2000 ; Tondreau, Morisset, 1998), les conditions dans lesquelles le transfert se déroule (Blanc, Perrier-Cornet, 1993) et les diverses étapes menant à celui-ci (Errington, 1999 ; St-Cyr et al., 1998), les stratégies d'établissement (Parent et al., op. cit. ; Perrier et al., 2004 ; Taylor et al., 1998 ; Tondreau, Morisset, op. cit. ; Tondreau et al., 2002) et les problématiques socio-organisationnelles familiales (Taylor, Norris, 2000). 
Dans les années 1990, Colson et Pineau (1991) ont proposé des indicateurs de détection de la difficulté financière des exploitations françaises en période d'établissement. Toutefois, au Québec, aucune recherche portant sur le lien entre la situation financière au moment du transfert et la performance post-transfert n'a jamais été présentée. La présente étude a comme objectif principal de découvrir les déterminants financiers et technico-économiques qui influencent la performance des exploitations agricoles à la suite du transfert intergénérationnel. Pour répondre à cet objectif, les résultats financiers de trois moments clés ont été observés : le montage financier permettant le transfert des avoirs, la situation de l'exploitation l'année du transfert et l'évolution de l'exploitation (figure 1). Ce cadre conceptuel, inspiré de celui proposé par Morris et al. (1997), permet de compléter les aspects organisationnels (le niveau de préparation des repreneurs, la nature des relations parmi les membres de la famille et les activités de planification et de contrôle) étudiés par cet auteur. Une étude parallèle à celle-ci (Gariépy, 2009) a démontré que les facteurs organisationnels (préparation à l'établissement, durée de la planification, accompagnement du cédant) sont explicatifs de la performance de l'exploitation, cinq ans après le transfert des avoirs. Bien qu'importants pour la performance de l'exploitation transférée, ces aspects ne seront pas traités à nouveau dans le présent article.

Cette étude propose de vérifier l'impact, en termes financiers et technico-économiques, de ces périodes sur la performance des entreprises cinq années après leur transfert. Pour y arriver, nous proposons d'amé- liorer un indicateur traditionnel de la performance, la marge de sécurité, afin de prendre en compte dans l'évaluation de la performance la capacité de l'exploitation : à rembourser ses emprunts, à renouveler ses immobilisations et à dégager un surplus.

Dans un premier temps, l'intérêt de l'évaluation de l'impact de ces trois périodes sur la performance post-transfert est présenté, suivi de la méthodologie utilisée et des résultats descriptifs de cette étude. Il est ensuite question des résultats obtenus par la régression linéaire multiple effectuée à partir des indicateurs financiers et technico-économiques sur la performance de 158 exploitations agricoles québécoises ainsi que des enseignements qu'il est possible d'en tirer.

\section{Les trois périodes financières clés}

Le processus de transfert d'exploitation inclut une passation (successeur/repreneur) à trois niveaux : les savoirs (Samson, 2004), les pouvoirs et les avoirs (Hugron, 1991 ; Samson, op. cit.). Dans les exploitations familiales, le transfert des savoirs se fait à partir de l'enfance et se poursuit durant plusieurs années. Le transfert des pouvoirs, nommé transfert de direction par Hugron (op. cit.), est en lui-même un processus de longue durée qui peut être défini en quatre étapes : l'incubation, le choix du successeur, le règne conjoint et le désengagement.

À la fin de la phase de règne conjoint entre le cédant et le repreneur, ce dernier devient le propriétaire majoritaire de l'exploitation et la dernière passation s'effectue : le transfert des avoirs. La réalisation de cette étape se fait à l'aide du montage financier qui est représenté par la combinaison des divers modes d'acquisition utilisés que sont le don, l'emprunt, les

\section{Figure 1. Cadre conceptuel des déterminants de la performance post-transfert}

\begin{tabular}{|lll}
\hline Pré-transfert & Année du transfert & Ṕvolution de l'entreprise \\
Montage financier & $\begin{array}{c}\text { Permance } \\
\text { post-transfert }\end{array}$ \\
St technico-économique & Source : les auteurs
\end{tabular}


subventions et les apports personnels. Au Québec, deux études, Perrier et al. (op. cit.) et St-Cyr et al. (op. cit.), ont été produites sur le financement de l'acquisition des fermes familiales. Leur conclusion similaire est que la combinaison des modes d'acquisition est une pratique courante et que les emprunts (bancaires ou aux parents) ainsi que les dons sont très importants. Dans la période précédant le transfert, les propriétaires (cédants et repreneurs) évaluent et choisissent le montage financier qui permettra, dans un premier temps, à l'exploitation de survivre, et dans un second temps, d'assurer aux cédants une retraite convenable. La combinaison des moyens financiers nécessaires à l'achat de la ferme est, à notre avis, un déterminant de la performance post-transfert étant donné son impact sur le taux d'endettement et par le fait même sur la capacité d'investissement des repreneurs. D'où la première hypothèse : H1. Le montage financier est un déterminant de la performance posttransfert

Le second moment financier clé est la situation de l'exploitation l'année du transfert. Avant de faire l'acquisition d'une exploitation, les acheteurs dressent un bilan indiquant la situation financière de celleci. Pour faire cette évaluation, deux types de ratios sont généralement utilisés : les indicateurs financiers et les indicateurs technico-économiques.

Pour ce qui est des indicateurs financiers, plusieurs ratios peuvent être utilisés tels que le fonds de roulement, la structure financière, l'effet de levier, l'autonomie financière, les rentabilités économique et financière. Trois éléments principaux semblent essentiels à évaluer afin de saisir la globalité de la situation financière. Tout d'abord, des indicateurs de liquidité qui indiquent la capacité d'une exploitation à subvenir à ses besoins de court terme (fonds de roulement). Ensuite, des mesures de moyen et long terme pour évaluer la capacité de l'en- treprise à faire face annuellement à ses obligations de même durée (taux d'endettement, capacité de remboursement, solde résiduel, rentabilité). Enfin, l'appréciation de la solvabilité (quantité et qualité des garanties) utilisée plus spécifiquement en cas de cessation volontaire ou contrainte de l'activité.

Par la suite, les indicateurs technico-économiques permettent de mesurer la capacité de l'exploitation à transformer de façon efficace les intrants en extrants et ce afin de générer une marge la plus élevée possible (Levallois, Perrier, 2002 ; Walsh, Anderson, 2006). La figure 2 illustre cette affirmation en schématisant les interrelations entre les produits, les charges et les indicateurs de l'efficacité technico-économique.

Le point de départ de l'efficacité technicoéconomique est la relation entre les produits ou, en termes financiers, le chiffre d'affaires (CA), et les charges d'exploitation. Cette relation mène à trois ratios :

- la productivité du capital, calculée en divisant les produits (CA) par les actifs immobilisés ;

- l'inverse du pourcentage de charges qui correspond aux produits (CA) divisés par les charges d'exploitation ;

- la marge brute de l'exploitation, obtenue en soustrayant les charges d'exploitation des produits (CA). En enlevant de cette marge brute les intérêts, les salaires et les amortissements, il est possible d'obtenir le résultat ou bénéfice net (hors impôt). Ce résultat (en lui additionnant les intérêts et les amortissements) est utilisé pour déterminer les capacités de remboursement (CDR) maximale et optimale (en lui additionnant uniquement les intérêts). La CDR maximale correspond au montant le plus élevé que l'exploitation peut rembourser en termes d'annuité. Cette CDR ne prend pas en compte le renouvellement des immobilisations contrairement à la CDR optimale. 
Lorsque les annuités sont enlevées de la $\mathrm{CDR}$, il reste un solde résiduel qui correspond au «coussin de sécurité » dont disposent les exploitations pour absorber une variation négative du CA et/ou autofinancer les nouveaux investissements. Le ratio entre le solde résiduel et le CA est nommé «marge de sécurité ». Exprimée en pourcentage, elle s'interprète de la façon suivante : une marge de sécurité de $5 \%$ signifie que l'exploitation peut subir une baisse de son chiffre d'affaires de $5 \%$ tout en continuant de rembourser ses annuités, si elle a été calculée avec la CDR maximale, ou encore de continuer de rembourser ses annuités et de renouveler ses immobilisations, si elle a été calculée avec la CDR optimale. Une variation plus grande du CA pourrait donc conduire l'entreprise à des difficultés financières.

L'état de santé financière de l'exploitation, l'année du transfert, est, selon nous, un déterminant de la performance post-transfert.
Même s'il n'est pas évident que le repreneur ait les mêmes compétences de gestionnaire que le cédant, il semble, a priori, logique de penser qu'une entreprise en bonne santé a plus de chance d'avoir de meilleures performances cinq ans plus tard. D'où la deuxième hypothèse : H2. La situation financière et technico-économique est un déterminant de la performance post-transfert.

La dernière période est celle post-transfert, qui permet de vérifier la qualité de la gestion et l'efficacité du nouveau repreneur. L'évolution durant les cinq années suivant le transfert, sous la direction du repreneur, autant au niveau financier que technico-économique influence également la performance finale. La troisième hypothèse pose donc que l'évolution de l'exploitation dans les années suivant le transfert, qui est fonction du profil, de la vision et des objectifs du repreneur, est un déterminant de la performance post-transfert. H3. L'évolution de l'entreprise est un déterminant de la performance post-transfert.

Figure 2. Schéma des interrelations entre les indicateurs technico-économiques

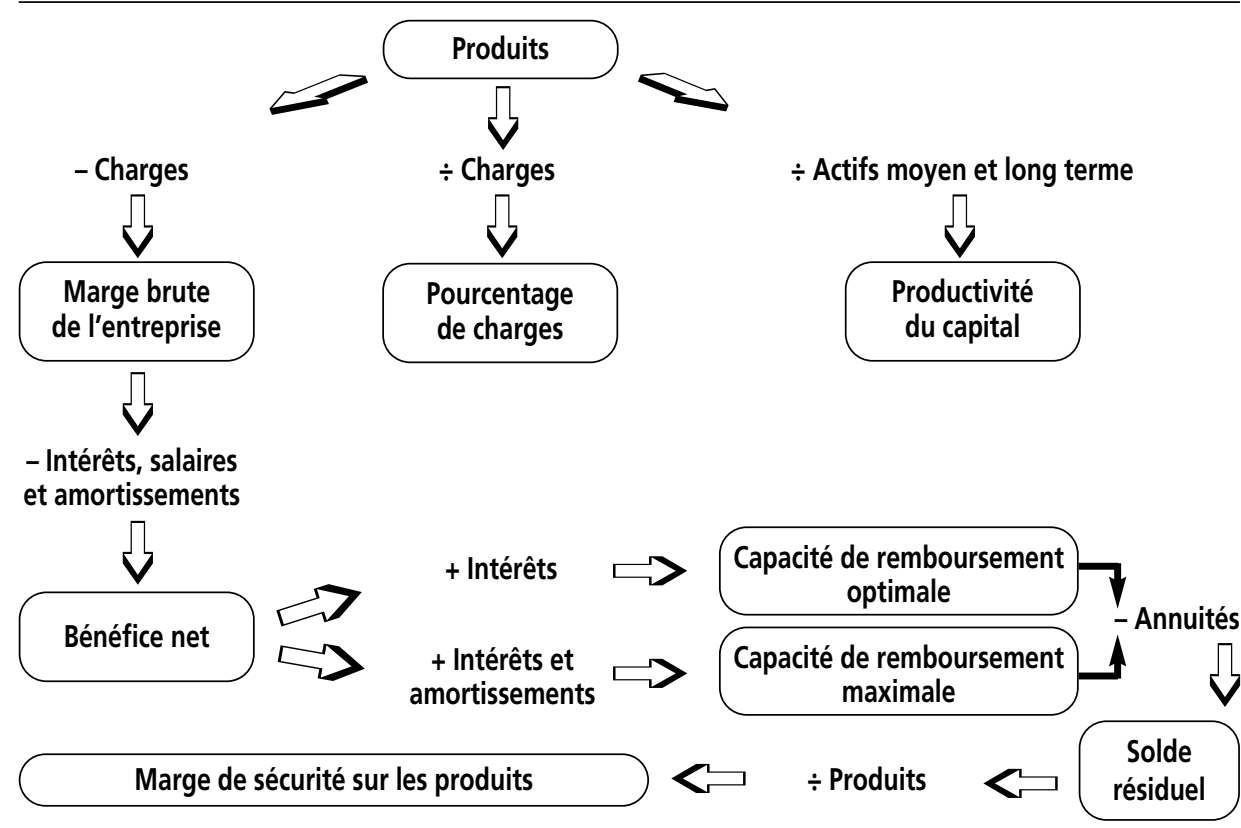

Source : les auteurs 


\section{Méthodologie de la recherche}

\section{1. Échantillon}

Cette recherche a été réalisée en collaboration avec la Financière agricole du Québec (FAQ). Cet organisme a comme mandat de stimuler les investissements et de protéger les revenus en vue de favoriser la réussite et la pérennité des exploitations agricoles québécoises. Les données comptables des clients de la FAQ répondant aux critères de sélection suivants ont été utilisées :

- exploitation transférée en 1999 ou en 2000. L'année du transfert des exploitations est évaluée par la présence d'un changement de propriétaire principal (individu possédant $50 \%$ des parts ou plus); - exploitation toujours existante en 2005; - présence à la FAQ des états financiers entre 1998 et 2004 ou entre 1999 et 2005.

La FAQ a extrait, selon ces critères de sélection, 207 exploitations. Par contre, 49 n'ont pas été utilisées dans l'analyse puisque les données se sont révélées incomplètes. Les données manquantes se trouvaient principalement dans les états financiers de 1998 et 1999 soit l'année avant le transfert et sans elles l'étude du montage financier était impossible. L'échantillon a donc été constitué des données de 158 exploitations agricoles familiales.

Cette base de données ne contenant pas ou peu d'informations socio-économiques ou organisationnelles, l'étude a été limitée aux aspects financiers. Même si la base de données contient des informations sur plus de 20000 fermes parmi les 30000 que compte le Québec, le critère d'échantillonnage (transfert, permanence de l'entreprise sur six ans) a éliminé bon nombre d'entreprises, et en particulier celles qui ont disparu de la base pendant la période pour différentes raisons (non fourniture des données, changement d'identifiant, cession ou démantèlement de l'entreprise).

Dans une analyse portant sur la viabilité des entreprises, les entreprises cédées ou démantelées devraient être incluses car potentiellement non viables. Dans cette analyse de la performance à terme de l'entreprise, la comparaison des plus et moins performantes donnera des pistes d'améliorations potentielles pour ces dernières.

Les données utilisées pour calculer les variables de la première période sont celles de 1998 ou 1999 ; pour la deuxième période ce sont les années 1999 ou 2000 et pour la dernière c'est la variation des données entre 1999 et 2004 ou 2000 et 2005.

\section{La performance des exploitations agricoles}

À la suite du transfert d'une exploitation, il existe une période critique de cinq années durant laquelle le transfert a le plus de chances d'échouer. Effectivement, entre 40 et $50 \%$ des firmes disparaissent durant cette période (Lank, 1992) et ce pour diverses raisons : manque de planification et/ou de compétences du repreneur, ou encore situations financières irrécupérables. L'évaluation de la performance de ces exploitations et de ses déterminants est ainsi nécessaire pour pouvoir assurer à l'avenir leur meilleure pérennité. Il est toutefois difficile de trouver un indicateur complet parmi ceux proposés dans la littérature. Pour nous un indicateur complet de la performance de l'exploitation doit prendre en considération autant la capacité de l'exploitation à rembourser ses emprunts, qu'à renouveler ses immobilisations ou à dégager un surplus.

Les indicateurs traditionnels tels que la rentabilité économique et financière ou encore les variations des ventes en volume, du chiffre d'affaires ou des parts de marché ne sont pas adaptés aux particularités des exploitations agricoles. Ces exploitations sont dans la majorité des cas de petites tailles, gérées et détenues par les membres d'une famille et par conséquent elles ne visent pas nécessairement un objectif d'augmentation de la valeur actionnariale ou de forte rentabilité des actifs (financiers et humains) investis par la famille. 
Le cycle de vie de l'exploitation est également à prendre en compte dans l'évaluation de sa performance. Dans la présente situation, les exploitations considérées ont dans tous les cas été transférées depuis cinq ans. Contrairement à une exploitation qui est dans une phase de stabilité de son cycle de vie, l'exploitation récemment transférée aura comme principal objectif financier le remboursement des emprunts (sur une durée conséquente) et le renouvellement des immobilisations. Ceci s'explique par l'important endettement des exploitations pour acheter la ferme et la nécessité de changer le matériel et la machinerie quand le besoin s'en fait sentir.

La présente étude propose une amélioration d'un indicateur traditionnel de la performance : la marge de sécurité, afin de permettre une analyse synthétique de l'état de l'exploitation. Tel qu'expliqué à la figure 2, la marge de sécurité sur les produits représente la capacité d'une exploitation à assumer une diminution des produits. Le choix de ce ratio est basé sur l'importance, pour une exploitation, d'être en mesure de faire face à une diminution de son $\mathrm{CA}$, sans se placer dans une situation de difficulté financière (solde résiduel négatif) qui entraînerait à terme la faillite. Le tableau 1 montre, en deuxième colonne, les étapes à suivre pour calculer la marge de sécurité sur les produits, conventionnellement utilisée par les institutions financières.

Cet indicateur manque toutefois de réalisme pour évaluer la performance des exploitations transférées pour deux principales raisons. Dans un premier temps, il ne prend pas en compte le renouvellement des immobilisations (que l'on approche au montant des amortissements) ce qui implique que les exploitations doivent nécessairement recourir à l'emprunt pour investir. Dans un second temps, des durées d'emprunts très contrastées ont été observées : certaines exploitations avaient une excellente marge de sécurité mais des emprunts sur 40 ans tandis que d'autres avaient une marge plus faible mais un financement court sur cinq ans. Des modifications ont donc été apportées ici afin de construire une marge de sécurité standardisée sur les produits (MSSP), présentée en troisième colonne du tableau.

Premièrement, la capacité de remboursement optimale plutôt que maximale a été utilisée. Ceci permet de prendre en compte la capacité de l'exploitation à remplacer ses immobilisations et pas seulement son potentiel à rembourser ses emprunts. Deuxièmement, la marge de sécurité a été standardisée par rapport à la durée des emprunts. Ceci permet d'éviter des situations où la marge de sécurité est acceptable mais où les remboursements d'emprunts s'étendent sur des périodes très longues (30 ans et plus). Dans le cas présent, la standardisation a été faite selon la durée moyenne des emprunts de toutes les exploitations soit environ 13 ans. Cette nouvelle donnée a été utilisée afin de calculer le capital devant être inclus dans les annuités et ainsi obtenir un solde résiduel standardisé.

Le tableau 2 présente une typologie basée sur la MSSP qui permet la comparaison entre les différents niveaux de performance. Les exploitations du groupe 1 ne sont pas en mesure de rembourser la totalité de leurs

Tableau 1. Calcul des marges de sécurité sur les produits

\begin{tabular}{l|l|l}
\multicolumn{1}{c|}{ Ratios } & Marge de sécurité sur les produits & $\begin{array}{l}\text { Marge de sécurité standardisée } \\
\text { sur les produits (MSSP) }\end{array}$ \\
\hline $\begin{array}{l}\text { Capacité de remboursement } \\
\text { (CDR) }\end{array}$ & $\begin{array}{l}\text { Produits - Charges (avant } \\
\text { amortissements et intérêts) }\end{array}$ & $\begin{array}{l}\text { Produits - Charges (avant intérêts } \\
\text { après amortissements) }\end{array}$ \\
\hline Solde résiduel & CDR maximale - Annuités & $\begin{array}{l}\text { CDR optimale - intérêts - (dette } \\
\text { long terme / durée moyenne des } \\
\text { emprunts) }\end{array}$ \\
\hline
\end{tabular}

Source : les auteurs 
RECHERCHES

Fanny LEPAGE, Jean-Pierre COUDERC, Jean-Philippe PERRIER, Diane PARENT

Tableau 2. Typologie des exploitations selon la MSSP

\begin{tabular}{c|c|c|c|c}
\hline Groupes & Fourchette de MSSP & Performance & Nombre d'exploitations & MSSP moyenne (\%) \\
\hline 1 & $<-8 \%$ & Mauvaise & 72 & $-24,03$ \\
\hline 2 & Entre $-8 \%$ et $0 \%$ & Acceptable & 31 & $-3,56$ \\
\hline 3 & Entre $0 \%$ et $8 \%$ & Bonne & 27 & 3,17 \\
\hline 4 & $>8 \%$ & Excellente & 28 & 14,88 \\
\hline Total & & & 158 & $-8,47$ \\
\hline
\end{tabular}

Source : les auteurs

annuités. Elles sont donc en mauvaise posture : incapables de faire face au risque économique et d'autofinancer le renouvellement des immobilisations. La conséquence de cette dernière incapacité est le recours à l'emprunt (augmentation des annuités) pour financer le renouvellement d'immobilisations (qui généralement n'entraîne pas d'augmentation de la productivité et des CDR), ce qui aggrave la situation et mine la pérennité de l'exploitation. Celles du groupe 2 ont la capacité de payer leur annuité mais ne couvrent pas la totalité de leurs amortissements, elles sont donc dans une position acceptable à court terme mais qui, à long terme, pourrait devenir critique et les faire basculer dans le groupe inférieur. Les exploitations du groupe 3 présentent un résultat intéressant puisque leurs produits peuvent varier (d'un maximum de $8 \%$ ) sans remettre en question le remboursement des emprunts ou le renouvellement des immobilisations Finalement, les exploitations du groupe 4 sont dans une excellente situation et peuvent assumer un niveau de risque élevé.

Le tableau 2 indique également le nombre d'exploitations présentes et la moyenne de MSSP de chaque groupe. Le principal constat est le nombre élevé d'exploitations qui se trouvent dans la plus mauvaise des situations : près de la moitié des exploitations (46\%), cinq ans après le transfert, ne sont pas en mesure de satisfaire, a minima, leurs obligations financières. Ceci se reflète dans la moyenne de la marge de sécurité standardisée $(-8,47 \%)$. La dispersion des résultats parmi les groupes permet de penser qu'une meilleure connaissance des déterminants de la performance post-transfert donnerait des pistes d'amélioration pour la majorité (ou presque) des entreprises.

\section{Résultats descriptifs}

Globalement au niveau du montage financier, deux constats sont particulièrement intéressants en ce qui concerne les résultats descriptifs qui sont inscrits dans le tableau 3 (noter, les groupes de MSSP 2 et 3 ont été regroupés pour mettre l'emphase sur les groupes 1 et 4 qui représentent les extrêmes de performance). Le premier est la répartition parmi les groupes du pourcentage d'exploitations données ${ }^{1}$ par le cédant au repreneur. Curieusement le groupe 1 est constitué à environ $30 \%$ d'exploitations données tandis que le groupe 4 en a seulement $11 \%$. Ce sont également ces dernières qui ont le plus emprunté pour acheter la ferme : ce montant est d'ailleurs croissant avec les groupes, passant de 143810 \$ pour le premier groupe à $370052 \$$ pour le dernier.

Le second constat émanant du montage financier est l'évaluation du prix de vente de l'exploitation : 0,84 \$ $\$$ de produits. Ce montant se rapproche certainement plus de la valeur économique des capitaux propres que de leur valeur marchande qui est évaluée, en 2001 , à 4,42 $\$$ de capitaux propres par dollar de produits selon les données de l'enquête financière sur les fermes produite par Statistique Canada (2007).

Ce dernier constat s'explique par le niveau de capitalisation élevé qui est l'une

1. Une exploitation est considérée comme « donnée » lorsque les dettes (emprunts, reconnaissance de dettes aux parents) n'augmentent pas consécutivement au transfert des avoirs. 
Tableau 3. Résultats descriptifs de la première période : montage financier

\begin{tabular}{l|c|c|c|c}
\hline Groupe MSSP & $\mathbf{1}$ & $\mathbf{2}$ et 3 & $\mathbf{4}$ & Moyenne \\
\hline Don total & $29,2 \%$ & $15,5 \%$ & $10,7 \%$ & $20.9 \%$ \\
\hline Emprunt pour l'achat & 143810 & 216575 & 370052 & 210615 \\
\hline Prix de l'achat/\$ de produit & 0,60 & 0,89 & 1,25 & 0,84 \\
\hline Valeur économique* $^{*}$ & 170225 & 235443 & 550442 & 318703 \\
\hline
\end{tabular}

Note : *La valeur économique des capitaux propres est ici approximée au montant d'un emprunt (20 ans, 6 \%) entraînant une annuité égale au solde résiduel.

Source : les auteurs

des caractéristiques des entreprises agricoles, en particulier au Canada où la terre est possédée par les producteurs à près de $90 \%$ et où les quotas de production (dans le lait, la volaille et les œufs) ont une valeur de marché importante. Puisque l'inflation a été et est encore élevée sur ces actifs, la productivité du capital diminue, creusant l'écart entre la valeur marchande des biens et leur valeur économique. Il paraît évident que cet écart malmène les valeurs patrimoniales, qui, normalement, conduisent à un transfert familial (avec un don élevé), au profit du démantèlement (réalisation des actifs pour en tirer l'entière valeur marchande).

L'écart entre la valeur économique et le prix de vente, beaucoup plus faible pour les trois premiers groupes que le quatrième, amène à penser que même si le groupe 4 rachète les parts de l'entreprise à un prix plus élevé, il les paie beaucoup moins que ce qu'il serait capable de payer et donc sa situation à terme devrait être encore meilleure que celle des premiers groupes. Il est donc logique de penser qu'il existe un lien entre le montage financier, la situation initiale et la situation finale.

Tel qu'indiqué au tableau 4, l'année du transfert, les exploitations sont, en moyenne, dans une situation financièrement « acceptable » : la marge de sécurité moyenne est de $9 \%$ et par conséquent les annuités peuvent donc être remboursées. Toutefois, le solde résiduel ne permet pas de procéder à la totalité des investissements annuels nécessaires : il représente uniquement $63 \%$ des amortissements. Seul le groupe 4 possède un solde résiduel suffisamment élevé pour renouveler ses immobilisations sans emprunter. Les exploitations les plus performantes, cinq ans après le transfert, sont dans une situation financière au moment du transfert « excellente » alors que les exploitations du groupe 1 ont une situation « acceptable».

Tableau 4. Résultats descriptifs de la deuxième période : année du transfert

\begin{tabular}{l|c|c|c|c}
\hline Groupe MSSP & $\mathbf{1}$ & $\mathbf{2}$ et 3 & $\mathbf{4}$ & Moyenne \\
\hline Marge de sécurité maximale & $6,16 \%$ & $8,40 \%$ & $16,19 \%$ & $9,05 \%$ \\
\hline Solde résiduel & 14841 & 20527 & 47990 & 22803 \\
\hline Amortissements & 32888 & 36101 & 43205 & 35896 \\
\hline Taux d'endettement & $77 \%$ & $72 \%$ & $81 \%$ & $76 \%$ \\
\hline Actif total & 672179 & 725023 & 672684 & 691667 \\
\hline Dettes & 517193 & 519104 & 542025 & 522295 \\
\hline Productivité du capital & 0,36 & 0,34 & 0,44 & 0,36 \\
\hline Produits (taille) & 240741 & 244487 & 296419 & 251983 \\
\hline Pourcentage de charges & $76 \%$ & $73 \%$ & $70 \%$ & $73 \%$ \\
\hline Marge économique & $10,76 \%$ & $12,25 \%$ & $15,41 \%$ & $12,26 \%$ \\
\hline
\end{tabular}


RECHERCHES

Fanny LEPAGE, Jean-Pierre COUDERC, Jean-Philippe PERRIER, Diane PARENT

Tableau 5. Résultats descriptifs (\%) de la troisième période : post-transfert

\begin{tabular}{l|c|c|c|c}
\hline Groupe MSSP & $\mathbf{1}$ & 2 et 3 & 4 & Moyenne \\
\hline Variation de la taille & 64 & 52 & 54 & 58 \\
\hline Variation de l'actif total & 56 & 42 & 46 & 49 \\
\hline Variation du passif total & 77 & 54 & 15 & 57 \\
\hline Variation du taux d'endettement & 13 & 9 & -21 & 6 \\
\hline Autofinancement & -6 & 6 & 74 & 11 \\
\hline Variation de la productivité du capital & 5 & 8 & 6 & 6 \\
\hline Variation du pourcentage de charges & 14 & -5 & -7 & 4 \\
\hline
\end{tabular}

Pour ce qui est du taux d'endettement (76 \%), il peut paraître très élevé, mais il est calculé sur des actifs à un coût d'acquisition déprécié. Généralement les financiers, pour apprécier les garanties, calculent le taux d'endettement sur la valeur marchande des actifs. Sur cette base, le taux d'endettement moyen des exploitations agricoles québécoises était de $24 \%$ en 2001 et de $27 \%$ en 2005 , toujours selon l'enquête financière sur les fermes de Statistique Canada.

Ensuite, en comparant les exploitations du groupe 1 et 4 , il est possible de constater que leurs actifs sont similaires mais que le groupe 4 a un taux d'endettement un plus élevé (soit $3 \%$ de plus) que celui du groupe 1. Le groupe 4 a également une productivité du capital et une taille plus élevées, tandis qu'il présente un pourcentage de charges inférieur. Ces écarts conduisent à une différence de presque $5 \%$ entre le groupe 1 et 4 sur la marge économique qui est obtenue par le ratio bénéfice net plus intérêts sur le chiffre d'affaires.

Selon les résultats descriptifs indiqués au tableau 5, dans les cinq ans suivant le transfert des avoirs, les exploitations, sous le règne de la relève, ont connu une croissance importante : de la taille, des actifs, mais, de façon inquiétante, aussi du passif (en moyenne plus importante que celle de l'actif). Le groupe des exploitations performantes n'a vu son passif augmenter que de $15 \%$ et son taux d'endettement se réduire de $21 \%$, contrairement au groupe 1 dont le passif a progressé de $77 \%$ et le taux d'en- dettement de $13 \%$. Il faut ici souligner que l'autofinancement, qui est le pourcentage des investissements réalisés sans recourir aux emprunts, est de $74 \%$, pour le groupe 4 et négatif pour le groupe 1 . Ceci est signe de problèmes graves pour le groupe 1 puisque les producteurs de ce groupe utilisent ce différentiel de passif pour financer des déficits d'opération.

Au niveau des résultats et de l'efficacité technico-économique en particulier, les exploitations du groupe 1 ont augmenté leur pourcentage de charges de $14 \%$ tandis que les exploitations du groupe 4 , qui pourtant avaient un pourcentage plus faible au moment du transfert, ont réussi à le réduire de $7 \%$. Des choix d'investissements plus productifs, moins d'investissements, une plus grande part d'autofinancement, une meilleure efficacité technico-économique sont les éléments composant la gestion des repreneurs dont les exploitations se retrouvent dans le groupe des plus performantes cinq années après le transfert.

La synthèse de cette brève analyse descriptive pourrait être :

- des exploitations peu performantes au moment du transfert sont transmises à un prix plus faible, qui est cohérent avec leur valeur économique. Leurs performances cinq ans plus tard se sont grandement détériorées suite au choix d'investissements moins productifs et du manque d'autofinancement dû à une gestion quotidienne (efficacité technico-économique) défaillante ; 
- des exploitations performantes au moment du transfert ont une valeur économique supérieure au montant nécessaire pour couvrir les besoins de retraite des cédants, et leurs performances cinq ans plus tard se sont encore améliorées.

Bien que ces conclusions ne dérogent en rien de la logique de gestion financière standard, il est inquiétant que le groupe d'exploitations en difficulté financière représente $46 \%$ de l'échantillon, ce qui questionne l'avenir du renouvellement des générations en agriculture, au moins au Québec. Elles confirment aussi l'intérêt d'approfondir les relations entre le montage financier, la situation initiale et la période post-transfert.

\section{Analyse statistique et résultats}

Pour vérifier nos hypothèses ( $\mathrm{H} 1, \mathrm{H} 2$ et H3) sur le lien entre les trois périodes et la performance post-transfert, une régression linéaire multiple a été effectuée sur la variable MSSP, construite pour synthétiser la performance de ces entreprises. Un ensemble de 15 variables continues, dont les calculs utilisés sont indiqués dans l'encadré 1, et 3 variables dichotomiques représentant l'aspect financier et technico-économique de chacune des périodes ont été utilisées comme variables indépendantes. Pour la période 1, les variables sont le montant emprunté pour acheter l'exploitation divisé par les produits et la présence (ou non) d'un don total de l'exploitation du cédant au repreneur. Pour la période 2 , le fonds de roulement, la marge économique, la marge de sécurité conventionnelle, l'endettement par dollar de produits, le pourcentage de charges, l'actif à long terme par dollar de produits, la taille, le secteur d'activité agricole et la région administrative ont été retenus. Finalement, ce sont les évolutions du fonds de roulement, de la marge économique, de l'endettement par dollar de produits, du pourcentage de charges, de l'actif long terme par dollar de produits et de la taille ainsi que l'autofinancement qui ont été choisies comme variables pertinentes de la période 3 .

\section{Encadré 1. Calculs des variables indépendantes}

Emprunt pour achat de la ferme par dollar de produits :

Variation des emprunts - achats d'immobilisation + billet au propriétaire

Produits

Ratio du fonds de roulement: Actif court terme

$\overline{\text { Passif court terme }}$

Marge économique : Bénéfice net + intérêts

Produits

Marge de sécurité sur les produits :

Capacité de remboursement - annuités

Produits

Pourcentage de charges :

Charges avant amortissements, intérêts et après rémunération du travail Produits

Actif long terme par dollar de produits : Actifs long terme

Produits

Évolution : tous les ratios d'évolution ont été calculés par la même formule soit

Ratio en 2004 ou 2005 - ratio en 1999 ou 2000

Ratio en 1999 ou 2000 
RECHERCHES

Fanny LEPAGE, Jean-Pierre COUDERC, Jean-Philippe PERRIER, Diane PARENT

Tableau 6. Résultats statistiques des déterminants de la performance post-transfert

\begin{tabular}{c|c|c|c|c|c|c|c}
\hline & $\begin{array}{c}\text { Coefficient } \\
(\beta)\end{array}$ & $\begin{array}{c}\text { Écart } \\
\text { type }(\beta)\end{array}$ & $\begin{array}{c}\text { Plus- } \\
\text { value }\end{array}$ & VIF & Élasticité & $\begin{array}{c}\text { Moyenne } \\
(\chi)\end{array}$ & $\begin{array}{c}\text { Écart } \\
\text { type }(\chi)\end{array}$ \\
\hline $\begin{array}{c}1 \text { - Pourcentage } \\
\text { de charges }\end{array}$ & $-0,43$ & 0,132 & 0,001 & 5,124 & 2,71 & 71,8805 & 16,89 \\
\hline $\begin{array}{c}\text { - Évolution de l'actif } \\
\text { long terme par } \\
\text { dollar de produits }\end{array}$ & $-12,07$ & 2,404 & 0,000 & 1,778 & 0,96 & 0,0380 & 0,53 \\
\hline $\begin{array}{c}3 \text { - Marge de sécurité } \\
\text { sur les produits }\end{array}$ & 0,22 & 0,085 & 0,012 & 3,703 & 0,39 & 7,1270 & 22,16 \\
\hline \begin{tabular}{c}
4 - Marge économique \\
\hline - Actif long terme par \\
dollar de produits
\end{tabular} & $-1,47$ & 8,516 & 0,009 & 3,013 & 0,33 & 0,1216 & 0,20 \\
\hline $\begin{array}{c}6-\text { Évolution } \\
\text { de la taille }\end{array}$ & 1,73 & 0,413 & 0,000 & 2,167 & 0,09 & 1,1018 & 3,99 \\
\hline $\begin{array}{c}7 \text { - Évolution du pour- } \\
\text { centage de charges }\end{array}$ & $-57,93$ & 4,621 & 0,000 & 2,171 & 0,08 & 0,0834 & 0,31 \\
\hline
\end{tabular}

Tous les postulats nécessaires à l'utilisation de la régression linéaire multiple ont été vérifiés. De la multicollinéarité a été détectée entre l'endettement par dollar de produits et les autres variables. Nous l'avons donc retiré de notre modèle. Le modèle final présente un $\mathrm{R}^{2}$ ajusté de 0.632 ce qui indique que les variables significatives de la régression permettent d'expliquer $63 \%$ des variations de la performance post-transfert. Le tableau 6 détaille ces variables significatives ainsi que leur coefficient non standardisé qui permet de déterminer si la variable a un impact positif ou négatif sur la performance (les autres variables et la constante du modèle ne sont pas incluses dans ce tableau afin de prioriser les variables significatives). Les variables sont ordonnées selon leur coefficient d'élasticité ${ }^{2}$ qui indique la varia-

2. Pour calculer ces coefficients, un ratio représentant la valeur des observations pour une variable divisée par la valeur des observations pour la marge de sécurité standardisée sur les produits a été calculé. Ceci nous permet d'obtenir un ratio pour chaque observation et pour chaque variable significative. Par la suite, le coefficient $\beta$ de chaque variable a été multiplié avec les ratios qui lui sont associés. Cette étape donne l'élasticité de chaque entreprise pour les sept variables. Finalement, la moyenne des élasticités individuelles pour chaque variable a été calculée puis inscrite au tableau 6 . tion de la variable dépendante suite à une variation de $1 \%$ de la variable indépendante (toutes les autres ne variant pas).

Contrairement à ce que laissait penser le cadre conceptuel, l'analyse de notre échantillon ne permet pas d'affirmer que le montage financier est en lien avec la performance post-transfert de l'exploitation : aucune variable de cette période n'est significative. Avant de conclure que le montage financier n'est pour rien dans les performances futures de l'exploitation, il convient de prendre en considération que généralement le transfert de l'exploitation familiale poursuit, au niveau financier, essentiellement deux objectifs (Eaton, op. cit.) : la pérennité de l'exploitation transférée et les revenus de retraite du cédant. Le prix de vente est donc un compromis entre ces deux objectifs contradictoires. En situation financièrement difficile, le transfert de l'exploitation donne la prépondérance au premier objectif. Si bien que le prix de vente va être fonction de la capacité de l'exploitation à « se payer » au moment du transfert. Il est donc logique de constater que c'est dans le groupe 1 qui est, au moment du transfert, en moins bonne situation financière (marge économique, solde résiduel, productivité du capital faible, pourcentage de charges élevé) que le pourcentage d'exploitations qui ont 
été données est le plus élevé ou que les emprunts pour l'achat de la ferme sont les plus faibles. De plus, dans le groupe 4, la valeur de vente très inférieure à la valeur économique laisse penser que les deux objectifs (pérennité et niveau de retraite) sont atteints.

À l'opposé, les exploitations plus performantes au moment du transfert peuvent (auto-) financer un prix de vente plus élevé. Donc, si le montage financier n'est pas en lien avec la performance post-transfert de l'exploitation, il est étroitement lié à la situation financière au moment du transfert qui elle-même explique, au moins en partie, la situation post-transfert. En situation de transfert, le prix se base sur la valeur économique de l'exploitation, valeur certes fonction des actifs et de leur productivité, mais surtout de l'efficacité technico-économique. Alors qu'en situation de démantèlement, c'est la valeur de marché des actifs, beaucoup plus élevée que la valeur économique, qui s'impose, indépendamment de leur productivité et de l'efficacité avec laquelle ils sont utilisés.

Cependant, le fait que le montage financier ne soit pas en lien avec la performance future de l'exploitation amène à se questionner sur la forme des aides à l'établissement consenties par le Gouvernement, en particulier la prime à l'établissement (jusqu'à 40000 \$ de capital). En effet, il est facile d'imaginer que cette aide, par l'intermédiaire du prix de vente, devienne une aide au cédant plutôt qu'à la relève.

La situation de l'exploitation l'année du transfert influence, en partie, la performance future de l'exploitation (4 variables). Le pourcentage de charges (1) qui reflète l'efficacité technico-économique de l'exploitation est la variable qui a le plus de poids sur la performance. Il s'agit d'une variable de court terme dans le sens où elle relève de décisions tactiques (quantité d'engrais, surface en céréales,...) plus facilement modifiables d'une année à l'autre. L'actif à long terme par dollar de produits (5) est aussi une variable significative qui a un impact négatif sur la situation à terme. Cette variable reflète d'une part la productivité des capitaux qui se retrouve dans le montant de produits et d'autre part le niveau de capitalisation auquel sont rattachées des charges fixes, en particulier les amortissements. Ces deux variables influencent, au moins partiellement, la marge économique (4) qui représente la capacité à rémunérer les capitaux totaux de l'exploitation, ainsi que la marge de sécurité (3) qui mesure la capacité de l'exploitation à honorer ses obligations financières de moyen-long terme et à faire face au risque économique.

En bref, ces variables indiquent que la performance à terme dépend de la santé technico-économique et financière de l'exploitation au moment du transfert. C'est au niveau des indicateurs technico-économiques que se situe le cœur de l'avenir du transfert : ils sont tous significatifs (pourcentage de charges, marge de sécurité et productivité des actifs à long terme). L'enjeu majeur de la performance se trouve donc dans la capacité du producteur à transformer ses intrants en extrants le plus efficacement possible et au moindre coût possible. La réduction des charges, et l'augmentation de la productivité du capital et de la marge de sécurité sont des éléments qui influencent positivement la performance.

Tout comme pour les variables du montage financier, celles portant sur une dimension organisationnelle (le secteur de production, la région administrative et la taille) ne sont pas significatives. La surreprésentation des fermes laitières dans cet échantillon (> $70 \%$ par rapport à $30 \%$ dans la population) rend probablement le secteur de production non significatif. La non-significativité de la région semble permettre de conclure que la performance n'est pas liée à la densité des réseaux et services agricoles sur un territoire, compensée, au moins partiellement, par le niveau de capitalisation (terre en particulier).

Les résultats amènent aussi un constat 
particulièrement intéressant sur la taille de l'exploitation. Il existe d'importants débats en agriculture sur la constante augmentation de la taille des entreprises. D'une part, le discours qui associe taille et performance en vertu des économies d'échelles. D'autre part, plusieurs spécialistes évoquent la taille des exploitations comme une difficulté pour la reprise. La présente recherche tend à démontrer que ce n'est pas la taille de l'exploitation au moment du transfert qui a un impact sur la post-performance mais plutôt, comme il en sera question dans la prochaine section, l'évolution de la taille.

La troisième et dernière période (l'évolution) de l'analyse représente l'impact de la gestion effectuée par la relève sur la performance de la ferme. Trois variables se sont révélées être significatives soit l'évolution de l'actif long terme, de la taille et du pourcentage de charges. L'augmentation de l'actif à long terme et du pourcentage de charges ont un impact négatif sur la performance tandis que l'augmentation de la taille a une influence positive. La relève doit tenter de restreindre ses achats d'actifs et ses charges tout en améliorant ses ventes. La gestion menée par le repreneur joue donc un rôle important sur la performance, au même titre qu'une entreprise bien gérée au moment du transfert.

Cette analyse permet de réfuter l'hypothèse $\mathrm{H} 1$ qui supposait le lien entre le montage financier et la performance posttransfert et de confirmer, en partie, les hypothèses $\mathrm{H} 2$ et $\mathrm{H} 3$ qui énonçaient la relation entre la situation de l'exploitation l'année de transfert ainsi que de l'évolution de l'entreprise à la suite du transfert et la performance post-transfert.

\section{Conclusion}

La démographie en agriculture est en pleine transformation avec l'augmentation constante de l'âge moyen des producteurs agricoles (Statistique Canada, 2006). Bien entendu, cette hausse implique l'arrivée imminente de l'âge de la retraite pour de nombreux producteurs. Par le fait même, ils auront à faire un choix difficile : soit le transfert de l'exploitation à une relève familiale, soit la vente à une personne hors cadre familial ou encore le démantèlement de l'exploitation. Pour ceux qui optent pour le transfert de l'exploitation à leur enfant, plusieurs embûches risquent de les faire échouer dans leur démarche : le peu de rentabilité en agriculture, la faiblesse des revenus de retraite (Vilain, op. cit.), et la hausse des valeurs des actifs agricoles (Levallois, op. cit.).

Ces facteurs auront eu raison de nombreux cas de transferts : taux d'échec de 40 à $50 \%$ des transferts en l'espace de 5 ans. Cette problématique préoccupe les gouvernements, les producteurs, les consommateurs, les divers intervenants en agriculture et les chercheurs. L'intérêt des recherches dans le domaine du transfert des exploitations agricoles est principalement axé sur les facteurs de succès et d'insuccès, les étapes menant au transfert et les stratégies d'établissement. Nous avons noté un important manque de connaissances au niveau de l'aspect financier du transfert et ce malgré une recherche exploratoire de Parent (op. cit.) qui dénote que les difficultés financières et le faible potentiel de revenu sont des facteurs d'insuccès à l'établissement.

Dans l'objectif de « combler ce manque d'informations », une régression linéaire multiple sur la performance post-transfert, synthétisée par la marge de sécurité standardisée, a été effectuée sur un échantillon de 158 fermes. Cette analyse a permis de faire ressortir sept variables significatives, les plus explicatives de la variation de la performance de l'exploitation cinq années après le transfert soient : le pourcentage de charges, l'évolution de l'actif long terme par dollar de produits, la marge de sécurité sur les produits, la marge économique, l'actif long terme par dollar de produits, l'évolution de la taille et l'évolution de pourcentage de charges. Quatre de ces variables mettent 
l'emphase sur la situation de l'exploitation au moment du transfert, trois portent sur l'évolution de l'exploitation après le transfert. Contrairement à une hypothèse initiale, le montage financier du transfert des avoirs n'est pas en lien avec la performance ultérieure de l'exploitation car il est, en fait, très conditionné par la situation financière de l'exploitation au moment du transfert.

Cette analyse financière ne répond pas à toutes les questions et le sujet nécessite des études complémentaires. Plusieurs pistes de recherche doivent être considérées.

Dans un premier temps, puisque les déterminants identifiés sont issus d'un échantillon d'exploitations « survivantes », il semble nécessaire pour enrichir les conclusions de la présente étude d'élaborer une nouvelle recherche concernant les exploitations qui n'ont pas survécu jusqu'à la fin des cinq premières années suivant la transmission. Une telle étude permettra d'évaluer si les variables significatives sont les mêmes dans la détermination de la performance menant à la viabilité et à la faillite de l'exploitation.

Une autre piste de recherche consiste à inclure dans notre échantillon des exploitations n'ayant pas été transférées. Une évaluation des éléments déterminant la performance selon le cycle de vie de l'exploitation pourrait ainsi être réalisée ainsi que l'identification des variables affectant la performance spécifique aux exploitations transférée.

\section{RÉFÉRENCES BIBLIOGRAPHIQUES}

Blanc M., Perrier Cornet P. (1993). Farm transfer and farm entry in the European Community. Sociologiaruralis, vol. 33 (4-5), p. 319-335.

Colson F., Pineau B. (1991). Les indicateurs de détection de la difficulté financière des exploitations en période d'installation. Économie rurale, $\mathrm{n}^{\circ} 206$, p. 57-63.

Eaton H. (1993). Farm Succession, Viability and Retirement: Ten Case Studies from Canterbury. MAF Policy Technical Paper 93/16. Wellington (Nouvelle-Zélande), Ministry of Agriculture and Fisheries, $58 \mathrm{p}$. Errington A. (1999). La transmission de l'exploitation agricole familiale entre générations: une étude comparative de l'Angleterre, de la France et du Canada. Communication préparée pour l'Assemblée annuelle de la Table pancandienne de la relève agricole. Régina, Alberta.

Gariépy M.-C. (2009). La pérennité de l'entreprise agricole et son lien avec le contexte organisationnel au moment $d u$ transfert des avoirs. Université Laval, Faculté des sciences de l'agriculture et de l'alimentation, Mémoire de maitrise.
Hugron P. (1991). L'exploitation familiale : modèle de réussite du processus successoral. Montréal, Institut de recherches politiques et Presses HEC, 155 p.

Lank A. (1992). Les exploitations familiales européennes : espèces en voie de disparition ou puissants acteurs économiques? Revue économique et sociale, $\mathrm{n}^{\circ} 3,11 \mathrm{p}$. Levallois R. (2003). L'endettement en agriculture: un risque ou une chance? Drummondville, Colloque de l'entrepreneur gestionnaire, Traget Laval.

Levallois R., Perrier J.-P. (2002). Gestion de l'exploitation agricole : Guide pratique. Saint-Foy, Agri-Gestion Laval.

Lobley M. (2007). Transfert de ferme : quelques résultats tirés de la recherche mondiale. Où nous étions. Où nous allons ? Conférence internationale sur le transfert de ferme, Ottawa, University of Exeter. Centre for rural Policy research. MAPAQ (2006). Portrait de la relève agricole établie. Québec.

Morris M., Allen J.-A., Avila R.-A. (1997). Correlates of success in family business transitions. Journal of Business Venturing, $\mathrm{n}^{\circ} 12$, p. 385-401. 
Parent D., Jean B., Simard M. (2000). Transmission et maintien de la ferme familiale : analyse des facteurs et stratégies d'établissement de la relève agricole familiale via les situations d'insuccès. Québec, Université Laval Agri-Gestion Laval. Perrier J.-P., Allard I., Parent D. (2004). Transfert de ferme et démantèlement au Québec : études de cas. Sainte-Foy, Traget Laval, Université Laval.

Samson A. (2004). Vos futurs leaders, les identifier, les former. Éditions Transcontinental Inc., Les Éditions de la fondation de l'entrepreneurship, Collection Grands Défis.

St-Cyr L., Richer F., Depuis J.-P. (1998). La relève agricole familiale au Québec: portrait et facteurs de succès de la relève établie. Montréal, École des hautes études commerciales, Groupe femmes, Gestion et exploitations.

Statistique Canada (2007) Enquête financière sur les fermes. Tableau 002-0064: bilan de l'agriculture canadienne et régionale.

Statistique Canada (2006). Recensement de l'agriculture de 2006. Données sur les exploitations et les exploitants agricoles, $\mathrm{n}^{\circ}$ 95-629-XWF au catalogue.
Taylor J.-E., Norris J.-E. (2000). Sibling Relationships, Fairness, and Conflict over Transfert of the Farm. Family Relations, vol. 49(3), p. 277-283.

Taylor J.-E., Norris J.-E., Howard, W.-H. (1998). Succession Patterns of Farmer and Successor in Canadian Farm Families. Rural Sociology, vol. 63(4), p. 553-573.

Tondreau J., Morisset M. (1998). La formation agricole de la relève en agriculture : nouveau regard sur la réalité des futurs agriculteurs et des futures agricultrices. Sainte-Foy, Université Laval, Groupe de recherche en économie et politique agricoles (GRÉPA).

Tondreau J., Parent D., Perrier J.-P. (2002). Transmettre la ferme familiale d'une génération à l'autre : situation au Québec et regard sur le monde. Québec, Université Laval, Agri-Gestion Laval.

Vilain F. (2004). La transmission des PME artisanales, commerciales, industrielles et de services. Conseil Économique et Social, $94 \mathrm{p}$.

Walsh G., Anderson J. (2006). Gestion de la ferme familiale transmise de génération en génération. Ottawa, Conseil canadien de la gestion d'exploitation agricole 110 . 